\title{
Design and analysis of a general data evaluation system based on social networks
}

\author{
Hongyan $\mathrm{Li}^{1,2}$, Xixi Mao ${ }^{3^{*}}$, Chunxue $\mathrm{Wu}^{3}$ and Fan Yang ${ }^{4^{*}}$
}

\begin{abstract}
A social network is a social structure with a set of social actors and other social interactions between actors. The social network supports a set of methods to explain the structure of whole social entities as well as a variety of theories in these structures. The study of these structures uses social network analysis to explain local and global patterns and examine network dynamics. The related economic data is important for our economic developments. We could analyze the economic data to explore the investments. In this paper, we focus on the design and analysis of automatic management systems to make a decision about the petroleum projects.

Petroleum is the lifeblood of economic development. So, we need an automatic and effective management system to make the mining investments of petroleum. Before deciding to invest in the petroleum project, the engineers will describe this project by providing the sufficient economic data. Based on this data, the decision can undertake professional analysis, to determine whether the project is feasible. To automate the manual process and overcome the traditional evaluation method, the BP (back propagation) neural network is applied in a petroleum project economic evaluation. This method opens up a new way for the evaluation of petroleum projects. In this article, we first introduce the application of BP neural network. Then, we introduce the principle and shortcoming of BP neural network. Based on it, we optimize the BP neural network. Secondly, according to the characteristics of petroleum projects, we obtain the economic evaluation indicators about petroleum projects, which are used as the input of the BP neural network. Thirdly, we propose the design of the BP neural network and select the smallest simulation error of the BP neural network as a forecasting model of petroleum projects. In order to improve the accuracy, the labeled training samples are selected for BP neural network training. Finally, based on extensive simulation experimental results, we demonstrate our scheme about the BP neural network of petroleum project evaluation is effective and has overperformance than traditional schemes in terms of convenience and accurate decision-making suggestions.
\end{abstract}

Keywords: Improved BP algorithm, Economic evaluation, Petroleum projects

\section{Introduction}

The petroleum industry is the lifeblood of the national economy and is also a high-risk industry. It has special significance to the development of the national economy. Petroleum development is mainly characterized by high risk, huge investment, long development cycle, high

\footnotetext{
*Correspondence: mxx@st.usst.edu.cn; sally01_yang@163.com

The former short paper is published in the conference: Xixi Mao, Naixue Xiong, Chunxue Wu, Rui Li, An Automatic and Effective Petroleum Economic Evaluation Scheme Based on BP Neural Networks, 2016 International Symposium on Information Technology Convergence (ISITC 2016) Shanghai, China, Oct. 14-15, 2016

${ }^{3}$ School of Optical-Electrical and Computer Engineering, University of Shanghai for Science and Technology, Shanghai, China

${ }^{4}$ Zhongnan University of Economics and Law, Wuhan, China

Full list of author information is available at the end of the article
}

technology, and complex influence. Before the petroleum project is decided to invest and develop, the relevant personnel will provide the economic data of the relevant project. Then, the data will be provided by the decision maker for professional analysis to determine whether the project is feasible. For the development of the petroleum project investments, investors often require very careful and reasonable assessments. Before investing in a petroleum project, the declarers need to finish the complete data to declare and summarize the petroleum project evaluation reports and the economic benefits of the graphical trend analysis. Then, policymakers will declare whether the project is worth to invest according to a filing submitted for this petroleum project. So as a leader in the petroleum industry investment to make the data of 
evaluation and decision is very important. But the final assessment of the process is done by people completely, who always make decisions about the inevitable result of a subjective judgment.

With the increasingly fierce competition in the international petroleum market, the petroleum company's business management model, which takes economic efficiency as the center and maximizing value as the goal and takes project management as the carrier, gradually penetrates into the petroleum development activities. The need for evaluation is more and more urgent. The use of scientific economic evaluation methods is a prerequisite for ensuring the objectivity of economic evaluation. The purpose of economic evaluation of petroleum projects is to improve the accuracy of petroleum development investment decisions, rationally and effectively use limited resources, and maximize the efficiency of petroleum development investment. It is an important means for investors to specify the project's economic rationality and investment profitability, as well as the decision-making basis for ensuring the development and investment benefits.

We know of the artificial neural network (ANN) [1-4], which has a very good solution in terms of nonlinear data processing, and its main function is to use a large number of nonlinear relationships between parallel processing to simulate many of the neural network learning processes. The independent study found the certain mathematical relationship of the nonlinear relation between input value and output value. So we can easily deal with a nonlinear problem through a trained neural network. And ANN is essentially a processing system based on the black-box principle, as long as we provide input values and pay attention to the results of the final output. In other words, we do not have to learn the study process of ANN.

Based on the above two methods of the industry and their advantages and disadvantages, there are many defects in order to avoid the traditional economic decision method [5]; we think to apply neural networks to the economic evaluation of petroleum is very feasible. In order to determine whether a petroleum project is economically feasible, we picked out the best representations of the petroleum economic evaluation index of the project as the improved ANN input values. And according to historical data on the previous petroleum project, as the training sample data for the improved ANN, a petroleum economic evaluation system based on artificial neural network is made to overcome the traditional decision-making and exclude subjectivity, with closer to human thinking mode, but a more scientific way to make a final project economic evaluation of the feasibility.

In this paper, our main contributions are as follows. Based on the defects of the standard BP (back propagation) neural network, the improved neural network is put forward. Based on the characteristics of petroleum projects, this article synthetically selects the economic evaluation indicators about petroleum projects. Apply the improved BP neural network to the economic evaluation and forecast the feasibility of the petroleum projects. The results of the simulation experiment will be explained to show the correctness and feasibility of the application.

The rest of this paper is organized as follows. Section 2 is about the introduction of application of the neural network. Section 3 briefly introduces the theories of BP neural network and the improved algorithm. Section 4 provides the selection of economic evaluation indicators of petroleum projects. Subsequently, the design of a BP neural network and the results of the simulation experiment are presented in Section 5. The summary of the application and the future work are included in Section 6.

\section{Related work}

The application of BP neural network in forecasting has been very mature. BP neural network is a multilayer feed forward network trained according to the error back propagation algorithm. It is one of the most widely used neural network models. The BP neural network can learn and store a large number of input-output pattern mappings without prior disclosure of mathematical equations describing such mappings. Its learning rule is to use the steepest descent method to continuously adjust the weights and thresholds of the network through back propagation so that the sum of squared errors of the network is minimized. The BP neural network is similar to other neural network models. The difference is that the transfer function of the BP neuron is a nonlinear function. The most commonly used function is the log-sigmoid or tan-sigmoid. The BP neural network (BPNN) is generally a multilayer neural network.

BP neural network's information processing capacity is determined by the input and output neurons' characteristics, the network structure, and the connection weights. The complexity of the neural network structure affects the performance of the neural network. In [6], a hybrid STLF method is proposed based on the improved ensemble empirical mode decomposition (IEEMD) and BP neural network. The remaining IMFs and residual are forecast by BPNN, and then, the forecasting results of each component are combined with BPNN to obtain the final predicted load series. In order to devise bus lines and make daily scheduling more precisely [7], back propagation (BP) neural network to predict bus traffic is used. As the time factor and meteorological factor are the two important factors which affect traffic, they did the data preprocessing for bus data in Guangzhou from August to December in 2014 and conducted several experiments to determine the network structure and parameters which are suitable for prediction of bus traffic. Through tenfold 
cross-validation experiments, the model is demonstrated being able to apply to bus traffic forecast.

Elons et al. [8] propose a hybrid method of stochastic population-based search algorithm (particle swarm optimization), and a gradient-based algorithm (back propagation) is used to train a multiplicative neural network. The proposed network topology and training algorithms have shown superiority on the traditional neural network for determining the optimal drilling path. The proposed system generated proposed drilling plans that achieved more than $88 \%$ drilling decision accuracy which is measured according to the actual drilling path. Predicting the stock price is considered the most challenging and important financial topic. Reference [9] proposes a hybrid ensemble model based on BP neural network and EEMD to predict FTSE100 closing price. There are five hybrid prediction models, EEMD-NN, EEMD-bagging-NN, EEMD-cross validation-NN, EEMD-CV-bagging-NN, and EEMD-NN-proposed method. Experimental result shows that EEMD-bagging-NN, EEMD-cross validation-NN, and EEMD-CV-bagging-NN models performance are a notch above EEMD-NN and significantly higher than the single$\mathrm{NN}$ model. And EEMD-NN-proposed method's prediction performance superiority is demonstrated compared with the all presented models and was feasible and effective in predicting the FTSE100 closing price. As a result of the significant performance of the proposed method, the method can be utilized to predict other financial time series data. Zhang et al. [10] consider a new hot-rolled strip thickness model prediction method based on extreme learning machine (ELM). The network uses live production data for training and testing, compared with the BP network prediction model. Simulation results show that the model can predict the thickness more quickly and accurately and is able to meet the needs of actual rolling production.

Reference [11] presents techniques based on the development of BP neural network and radial basis function (RBF) neural network models, for modeling and predicting the daily network traffic at Universities East Kalimantan, Indonesia. The predicting daily network traffic usage is a very important issue in the service activities of the university. In addition, reference [12] proposes a new hybrid model that combines wavelet analysis (WA) and BP neural network called the wavelet neural network (WNN) model and applied for runoff time series prediction. BP network is selected as the neural network. The model is trained and tested by the year runoff time series of Tangnaihai Station located in Yellow River upper stream from 1956 to 2008. The performance of forecasting accuracy of the WNN model is relatively high compared to the traditional approach. And reference [13] adopts artificial neural network (ANN) to predict the human blood pressure. To extract the information of human emotion using Facebook, a vector model is applied here. The textual data is classified by a vector model, and ANN is used to predict human blood pressure. The outcome shows that ANN can be prosperously applied for prediction of BP through primary emotion. A new improved prediction method [14] which does not rely on any basic prediction methods is proposed based on analysis of a traditional wind power prediction procedure and in terms of a strategy on how to use a basic prediction method in wind power prediction procedure. Taking back propagation (BP) neural network as a basic prediction method, the proposed prediction method is validated by output power prediction of a real wind farm.

Wang et al. [15] add gray theory to BP neural network for predicting eggs' Haff value and evaluating freshness degree of eggs. The system automatically distinguished the egg shell color first, then the egg fresh degree can be predicted. Article [16] proposes that a preprocessing procedure on dense type of data, such as K-means, is presented using the error back propagation algorithm to predict share price. So appropriate pretreatment techniques of data could make the neural network execute more effectively to accept intensive data. In [17] has a research on movie box office forecasting based on a BP neural network. NAR [18] predicts a clearness index that is used to forecast global solar radiations. The NAR model is based on the feed forward multilayer perception model with two inputs and one output. Reference [19] presents a BP neural net real-time data forecasting model which is suitable for the home environment by using the correlation between the indoor temperature, outdoor humidity, and indoor humidity. According to problems of petroleum price prediction and the feasibility of petroleum price prediction model, the improved BP model [20] for petroleum price prediction is proposed. The hybrid training algorithm is combined with the improved particle swarm optimization and $\mathrm{BP}$ algorithm, while the improved PSO-BP ANN model is developed and trained by the hybrid algorithm based on improved PSO and BP algorithm. In [21], the ice thickness in Bohai Sea is predicted using the hybrid neural network model, and a good fitness is revealed between the prediction and practical values.

And the economic forecasting methods are generally divided into qualitative forecast method and prediction method. The first kinds of methods are expert investigation method and the survey method. The survey approach is used for consumers and producers to investigate their opinions or intentions for future development, considering their psychological factors. The second types of methods are time series method, index analysis, and factor analysis. Various prediction methods have their own scope of application, and the advantages and disadvantages can combine with each other, in order to test and supplement. Choose 
prediction method, starting from the characteristics of forecasting object, according to the purpose and requirements of forecast, which holds the data situation and predicts the comparison of cost and benefit factors synthetically considered.

\section{The mathematical description and optimization of BP neural network}

\subsection{Description of a three-layer neural network}

The BP neural network is the multilayer feedback neural network, which has three layers: input layer, hidden layer, and output layer. BP neural network have one or more hidden layers. The typical BP neural network structure is as shown in Fig. 1.

If the output values do not agree with the excepted values, the BP neural network can gradually adjust the connection weights and thresholds between neurons by back propagation of errors between the real output and excepted output. The process of learning can stop until the error value is in the range of the acceptable. The BP neural network will be close to a function by repeatedly studying the training samples with excepted values [21-26]. In other words, the learning process can finish any nonlinear mapping from $n$-dimension space of input to m-dimension space of output through adjusting the thresholds and weights to meet excepted values. Then, the trained neural network can be used for economic evaluation of petroleum projects. The BP algorithm process is shown below.

Step 1: Initialize the data. And the BP neural network obtains the input and desired output variables.

Step 2: The BP neural network obtains the output values through the hidden layer and output layer calculating the input values. Then, output errors are calculated by the difference of the expected and the actual values.

Step 3: . Whether the error values are all in the expected range. If output errors are judged that meet all requirements of prospective errors, then skip to step 5. if not, skip to step 4.

Step 4: When the calculated errors are too large, according to error gradient descent method to push the cumulative values to the BP neural network and jump into step 2.

Step 5: End the learning process of neural network. At that time the neural network has been a good way to learn.

From the mathematical analysis of BP neural network model, $x_{1} \sim x_{n}$ are the input signals from other neurons, $w_{\mathrm{ij}}$ represents the weight of the connection with the two neurons in the adjacent layer, and $\theta$ means a threshold. When the nodes of neurons in input layer receive signals $\left(x_{1} \sim x_{n}\right)$, the value of $n e t_{i}$ can be calculated by function (1).

$$
\text { net }_{i}=\sum_{j=1}^{n} w_{i j} x_{i}-\theta .
$$

And the relationship between input and output is expressed as formula (2).The output value $\left(q_{\mathrm{i}}\right)$ is produced by activation function and is passed to the next node of neurons in adjacent layer. And so on to get the final results.

$$
q_{i}=f\left(\text { net }_{i}\right) .
$$

The BP neural network selects the sign polarity sigmoid function (hyperbolic tangent function) as activation function, formula is as follows:

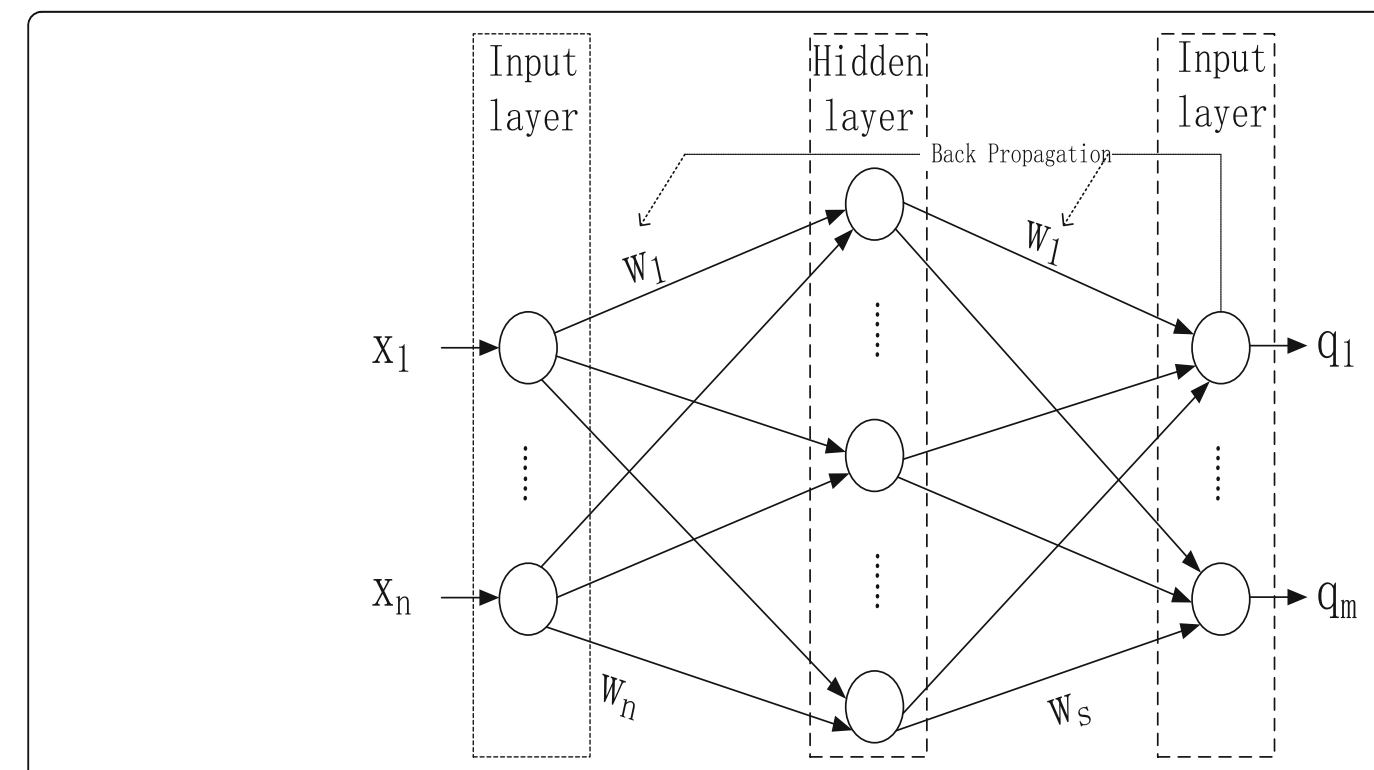

Fig. 1 The structure of a three-layer BP neural network 


$$
f(x)=\frac{1}{1+e^{-x}} .
$$

The final result will be acquired by adjusting the connection weights and thresholds. We must ensure that the error between excepted value and $q_{i}$ is in a reasonable range. Then, the error formula as formula (4).

$$
E=\sum_{P=1}^{P} E_{p}=\frac{1}{2} \sum_{p=1}^{P} \sum_{j=1}^{m}\left(t_{p j}-q_{p j}\right)^{2}
$$

If there are $\mathrm{P}$ groups of data samples, $E_{p}$ represents the first $\mathrm{p}$ sample error, $t_{p j}$ is the ideal output value for a neural network. And in the standard BP algorithm, the weight adjustment formula is as follows:

$$
\Delta w=-\eta \frac{\partial E}{\partial W}
$$

So the learning rate $\eta$ is set value in advance before learning. And we have no theoretical guidance, the value of learning rate is general scope in $[0,1]$. This kind of practice greatly affects the rate of convergence of the $\mathrm{BP}$ algorithm. If the learning rate initially is set too large. It is possible to improve the speed of convergence, but will lead to oscillation phenomenon, or even divergence. On the contrary, the vector is too small which can guarantee the stability of training convergence, but the learning speed will be slow. Therefore, a fixed vector could not be very well suitable for network of the whole learning process.

\subsection{The optimization of BP neural network}

Although the BP neural network application is already very mature itself, it still has a lot of defects. Because the BP algorithm in essence used the gradient descent method, and it is used to optimize the objective functions which are very complicated. Therefore, the error curved has flat regions. In these regions, error gradient changes smaller. Even if the adjustment of the weights is large, the error is still falling slowly, which makes the training process almost to a halt. The BP neural network training begins with a starting point for the tangent gradually reaching the minimum error. For complex network, the error function is the multidimensional space curved surface. Thus, during the process of the training, the BP neural network may be a local minimum value of a dell area, from this point to the direction of changes which all make error increases, so that training cannot jump out the local minimum value. Structural parameters (including the number of hidden layers, hidden layer neurons' number) are lack of theoretical guidance. We often can choose the value of parameters according to our experience. Under normal circumstances, the poor ability of trainings always has poor ability of generalization, and to some extent, with the increase of trainings, the generalization ability also will be improved. But there is a limit to this trend, when this limit is reached, the phenomenon will be coming, and with the increase of training ability, the generalization ability declines.

Aiming at these disadvantages and combining some optimization schemes, we want to joint optimization of BP neural network and then use the improved BP neural network in petroleum project economic evaluation.

First is the optimization of learning rate. Unlike previous plans, the learning rate is constant. We choose the dynamic adjustment of the adaptive learning rate. The main idea is that learning rate will change along with the difference of errors. When the change of error is smaller, we let the next learning rate become large; but when the error change is bigger, we reduce the learning rate in next step which can speed up the convergence rate of BP neural network during its learning process. The main modification formula is as follows:

$$
\eta_{t}=\eta_{(t-1)}(1-\alpha \Delta E), \Delta E=\left(E_{t}-E_{(t-1)}\right)
$$

Among the formula, $\eta_{t}$ and $\eta_{(t-1)}$ represent the learning rate in time period $t$ and $t-1 . \Delta E$ is the change of the error. $\Delta E$ is used to control the value of learning rate $\eta . \alpha$ is a stable factor, and generally, the scope of $\alpha$ is 0 . $01 \sim 0.03$, which is mainly used to ensure the stability of the algorithm by controlling the value of $\Delta E$.

For activation function, we use an improved Sigmoid function as formula (7).

$$
f(x)=\frac{a}{\left(1+e^{-x}\right)}, a \text { is constant }
$$

in which, $a$ will be the parameters of the BP neural network and also needed to keep the learning adjustment amount as neural network parameters.

Based on the traditional prediction methods, in order to avoid the potential disadvantages, the BP neural network is applied in predicting oil project feasibility. And now, we can use the improved neural network to do this. Making the neural network to predict oil economy has better persuasion and accuracy; the basic data, initially written from a set of oil economic evaluation systems, are extracted from these projects as the neural network experiment test data. So the method, with the advantages combined and the two shortcomings eliminated, will be better applied to the practical application.

\section{The indicator system of economic evaluation of petroleum projects}

Economic indicators are conducive to reflect the economic benefits of petroleum projects. Because of the complexity and particularity of petroleum projects, the 
single evaluation indicator is unable to give the comprehensive evaluation for the projects. So according to references [27-30], multiple interconnected and relatively independent evaluation indicators are to be adopted to constitute the petroleum project economic evaluation indicator systems. Figure 2 contains six indicators: net present value (NPV), net present value rate (NPVR), return on investment (ROI), Internet rate of return (IRR), present cost $(\mathrm{PC})$, and dynamic investment payback period (PT).

According to references [31-34], the economic evaluation indicators are divided into two categories. Determining whether a project is feasible, the indicators of net present value (NPV) will be first considered. NPV shows the difference between the cash inflows and outflows. The NVP is greater than or equal to zero, which means the project is feasible and can reach or exceed the benchmark yield. This project can bring profits to investors. Habitually, in comparing multiple project schemes, the project with the maximum NPV is worthy to invest. The evaluation criteria of the NPVR is the same as that of the NPV. Rather than the NPV, NPVR considers the size of the initial investment, which presents the ratio of NPV and the total investment. ROI refers to the unit investment profits. The larger the value of $\mathrm{ROI}$ is, the greater the project is. However, ROI is a static indicator, and the project is feasible when the value of ROI of this project exceeds the value of ROI. Internal rate of return (IRR), one of the important economic indicators, is defined as the discount rate when the NPV is zero. It means that the cash inflow just completely offsets the cash outflow and also produces on average IRR income level per year throughout the project life period. The chosen indicators must consider the cost of the project, so choose the indicator of present cost (PC) and dynamic investment payback period (PT).
PT is just an auxiliary indicator which presents the time limit when the cumulative profits equal zero. Compared with the static payback period, this economic indicator considers the time value of funds. Six selected indicators are designed to be the neurons' number of input layer.

\section{Economic evaluation of petroleum projects based on BP neural networks/methods}

\subsection{Normalization of economic evaluation}

The six selected indicators which predict the economic condition of the petroleum projects are used as input data of BP neural network. However, these data do not have the commensurability. It means that the unit of measurement is not the same. For example, some unit is percentage, and some is time for the unit. These data cannot be compared. The value of cost and profits about petroleum projects are so large for a BP neural network, which results in convergence of the BP neural network being slow and deviation being bigger. In a simulation experiment, we must take the activation function domain into consideration. The data is too large or too small which will influence the effect of a network learning. In order to solve these problems, generally, data preprocessing before the training sample is very important and data normalization can make BP neural networks stronger. Given the descriptions of paper [35-37], adopt min-max normalization as the data normalization method. But there are the two categories of the economic indicators. The values of cost indicators are as small as possible for the petroleum projects. On the contrary, if the values of the remaining indicators are greater, the petroleum projects bring more benefits for the investors. Therefore, suppose there are $n$ training sample and each training sample contains six values of economic indicators. The six values of economic indicators are divided into two types will be normalized by the following formula and limit in a $[0,1]$ interval.

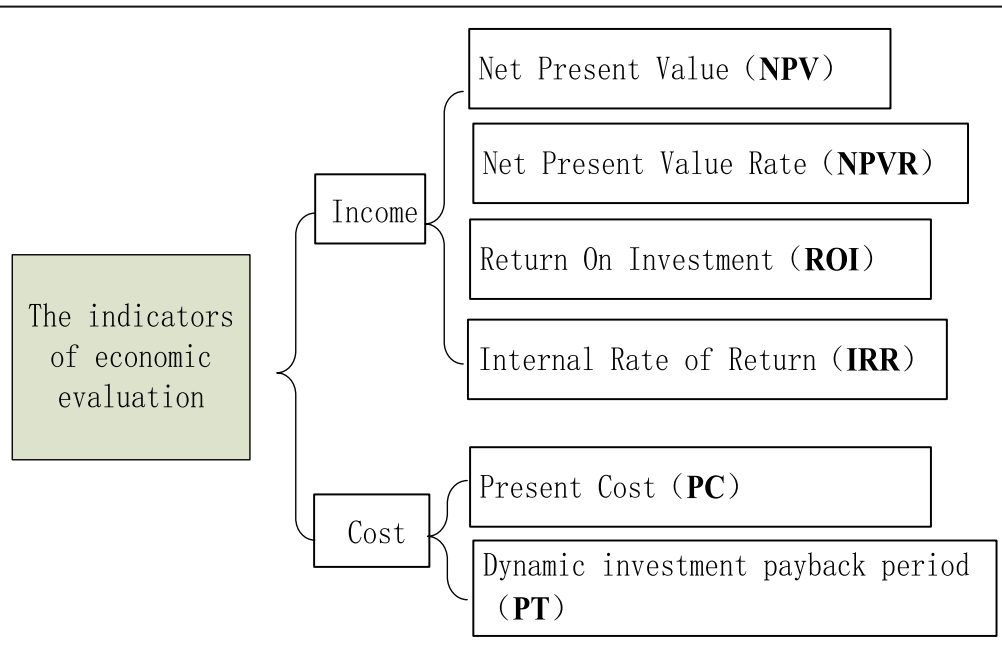

Fig. 2 The indicator system of economic evaluation 
Table $1 \mathrm{~A}$ part of the training samples

\begin{tabular}{llllllll}
\hline & NPV & NPVR & ROI & IRR & PC & PT & ex \\
\hline 1 & 474 & 13.25 & 9.66 & 12.65 & 6052.36 & 17.1 & 1 \\
2 & 148.22 & 26.2 & 18 & 12.25 & 400 & 5.59 & 1 \\
3 & -63.54 & -0.2 & 0.4 & 9.06 & 105.9 & 19.5 & 0.5 \\
4 & -136.5 & -1.3 & 0.2 & 8.22 & 300.28 & 10.25 & 0.5 \\
\hline
\end{tabular}

(1) If it belongs to the income indicator, including the net present value (NPV), net present value rate (NPVR), return on investment (ROI), and Internet rate of return (IRR), the value of it can be normalized by formula (4).

$y_{i j}=\frac{x_{i j}-\min x_{i}}{\max x_{i}-\min x_{i}} \quad i \in[1, n], j \in[1,6]$.

(2) If it belongs to the cost indicator, including the present cost $(\mathrm{PC})$ and dynamic investment payback period $(\mathrm{P})$, the value of it can be normalized by formula (5).

$$
y_{i j}=\frac{\operatorname{man} x_{i}-x_{i j}}{\max x_{i}-\min x_{i}} \quad i \in[1, n], j \in[1,6] .
$$

Among them, $x_{i j}$ is the original value of $j$ th indicators belonging to the $i$ th indicators group. $y_{i j}$, the output value, is obtained by the normalization of $x_{i j}$. $\max x_{i}$ and $\min x_{i}$ respectively represent the maximum and minimum values of the $i^{\text {th }}$ set of training samples.

\subsection{The design of BP neural networks}

The process of economic evaluation of petroleum projects by using BP neural network can be converted into

Table $\mathbf{2}$ The error values of each training

\begin{tabular}{ll}
\hline Number of neurons & Error value \\
\hline 4 & $2.44926232751162 \mathrm{e}-06$ \\
5 & $5.25642931493911 \mathrm{e}-07$ \\
6 & $2.65986047041910 \mathrm{e}-08$ \\
7 & $7.51942240950000 \mathrm{e}-09$ \\
8 & $3.08896072313119 \mathrm{e}-12$ \\
9 & $7.56619334523776 \mathrm{e}-10$ \\
10 & $2.33945409351388 \mathrm{e}-09$ \\
11 & $7.62970941440424 \mathrm{e}-09$ \\
12 & $4.09189402252038 \mathrm{e}-06$ \\
13 & $2.83784044194159 \mathrm{e}-08$ \\
\hline
\end{tabular}

nonlinear mapping between the selected economic indicators and the evaluation results. The BP neural network is designed to a three-layer structure. According to the number of economic evaluation indicators, the number of neurons of input layer is six, which is decided by the six aforementioned indicators [38, 39].

Common BP neural networks have only one hidden layer that can be enough to map from the $n$-dimension to the $m$-dimension. But it is difficult to determine a suitable number of neurons of a hidden layer. Too less or too large number of neurons will affect the network performance. In a general way, the number of neurons is determined by a trail-and-error method. Reference formula (6) sets up a series of the number of hidden layer nodes. Different training errors can be obtained by BP neural network training. Among them, we let different numbers of hidden nodes as the dynamic parameters of BP neural networks [40-42] and select the smallest simulation error of the BP neural network as a forecasting model of petroleum projects. Then, we will confirm

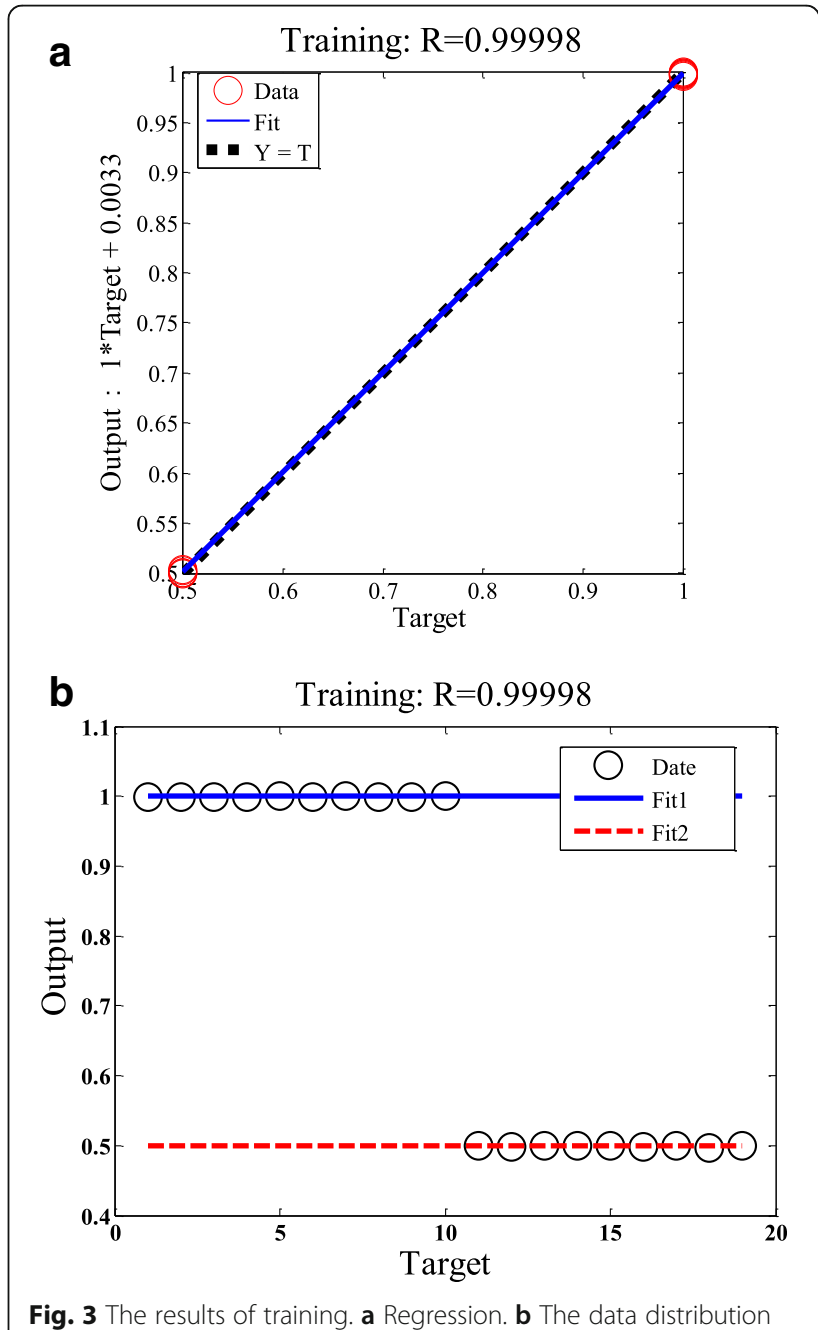




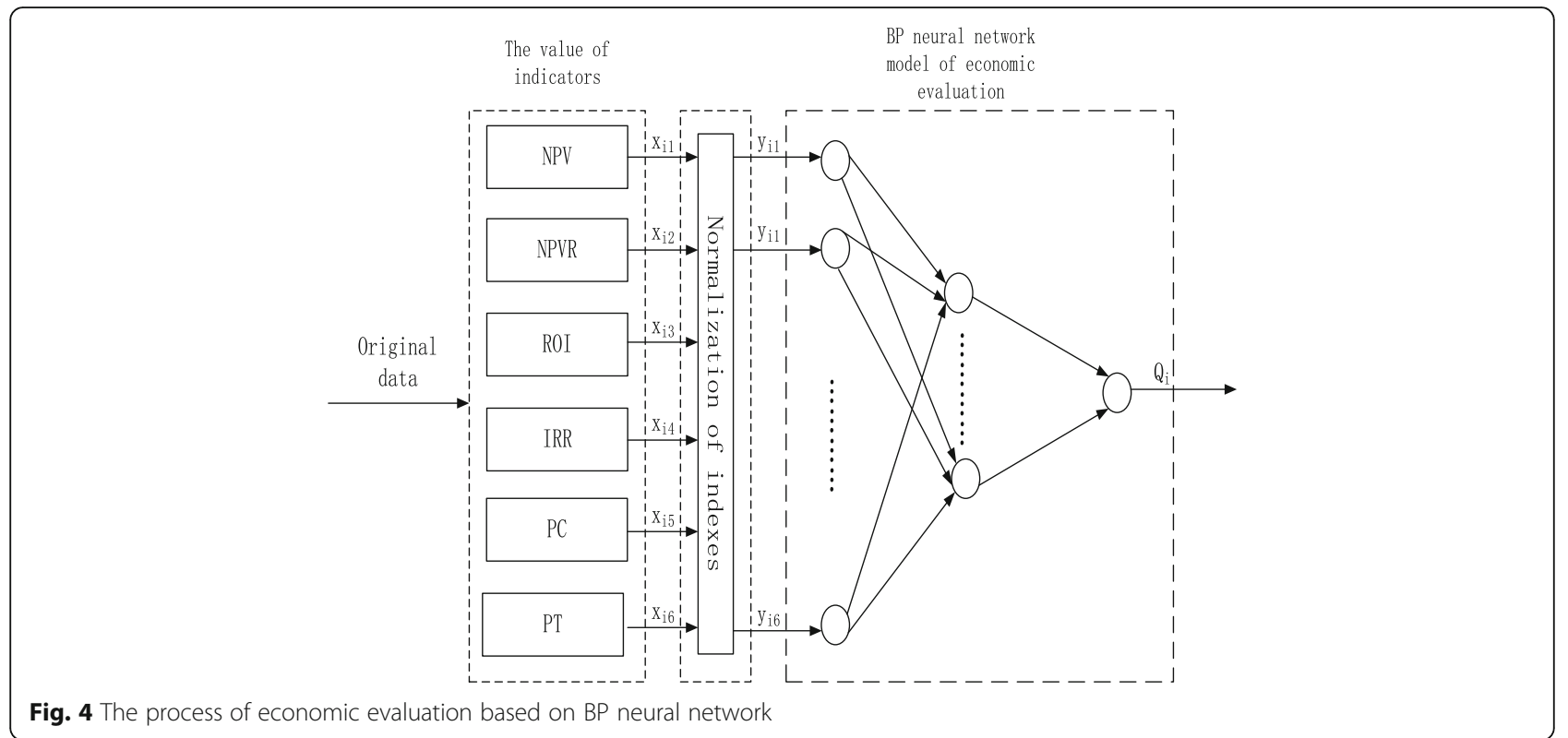

the number of hidden nodes and which training $\mathrm{BP}$ neural network has the minimum error.

$$
m=\sqrt{n+l}+\alpha
$$

where $m$ is the number of neurons of a hidden layer, $n$ represents the number of neurons of an input layer, and $l$ is the number of neurons of an output layer. $\alpha$ is a constant value, ranging from 1 to 10 . Based on the above definition, $n$ is equal to 6 . And the number of neurons of an output layer is 1 . And based on the range of $\alpha$, we can get the scope of $m$, which ranges from 4 to 13 . The final results of the economic evaluation were divided into two categories, feasible and infeasible.

As shown in Table 1, there is a part of a training sample. The excepted output value (ex) of BP neural network is 1 , which means the petroleum projects are feasible and profitable. When that value is equal to 0.5 , we should give up the petroleum projects because of no profit. From Table 2, through training a large amount of sample data, it is concluded that the number of neurons of a hidden layer is 8 . When the number of neurons is 8 , the error value is the smallest. And the training results are as those in Fig. 3, which can show us that the final data mapping is very successful, infinitely close to 0.5 and 1. The trained BP neural network can directly tell you whether the projects are feasible.

\subsection{The processes and results of economic evaluation based on BP neural network}

As shown in Fig. 4, we are able to understand the process of economic evaluation of petroleum projects based on BP neural network. The users can input the original data about the petroleum project, such as basic data, cost, business tax, and financing options. By submitting and calculating these data, the application will generate the petroleum projects' financial statements, which preliminarily reflect the profit values of petroleum projects. According to the basic calculation, the computer can carry an uncertainty analysis containing breakeven analysis and sensitivity analysis. Then, the more detailed financial statements and line chart will be used to improve the reliability of investment decisions. Finally, the final results, which represent the feasibilities of petroleum projects, are given to the decision makers.

For example, the dataset of economic indicators about some petroleum projects are listed in Table 3. The result value of number 1 , i.e., $Q_{1}$ is approximately equal to 1 , which means that this petroleum project is worth investing. And we also can analyze the testing data. Investors just spend 10 years recovering the cost and the shareholders earn \$2.04 million profits. The value of IRR is greater than $8 \%$, high investment recycling ability. Obviously, it is a

Table 3 The results of the testing data

\begin{tabular}{llll}
\hline & 1 & 2 & 3 \\
\hline NPV & 204 & -31.06 & 0.4 \\
NPVR & 456.76 & -5.89 & 3.8 \\
ROI & 13.2 & 50.4 & 4.89 \\
IRR & 21.36 & 0.12 & 2.1 \\
PC & 12.25 & 4.22 & 19.8 \\
PT & 10.33 & 11.5 & 20.93 \\
$Q_{i}$ & 0.999373314484295 & 0.500479758344815 & 0.497529927042037 \\
\hline
\end{tabular}


worthwhile investment project, which is consistent with the prediction result.

From the second column of Table 3, NPV, the most important economic indicator, is less than zero, which means this project fails to reach predetermined yield levels. And the time of cost-recovering is too long. So the value of $Q_{2}$ is closer to 0.5 . This project is a typical infeasible petroleum project. And the value of $Q_{3}$ is also closer to 0.5 . Based on an analysis of instance data, although the value of NPV is greater than 0 , a huge amount of investment, low profit, and long payback period of investment all means that project is not a worthwhile investment project. The result of the data analysis is consistent with the outcome given by the BP neural network.

\section{Conclusions}

The improved BP neural network, which was applied to economic evaluation of petroleum projects, is effective and suitable, and overcomes the defects and limitation of traditional assessments. The accuracy of the precision depends on the integrity of the training data. And we are through the application of petroleum economic evaluation to get a large number of real data. From the results of training experiments, BP neural network was trained by a large number of real data about the petroleum projects. Simulation results around the two lines: $y=0.1$ and $y=0$. 5. It can accurately describe the nonlinear relationship between the six indicators representing the economic benefits of petroleum projects and the feasibility of the project. A mature neural network can more accurately provide leaders the judgment of petroleum projects and save the human resource.

Of course, BP neural network also has its defects. There is no complete theoretical guidance for the confirmation of the number of hidden layers and their number of nodes. For the future work in this article, although the BP neural network can reasonably predict the feasibilities of the petroleum projects, there still exist some problems. Deep learning will be introduced to predict its economic benefits to make prediction more accurate. In order to improve the accuracy of prediction, the choice of the training samples and the economic indicators remain to be further perfected. Or through independent learning and removing the sample label, let the neural network take the initiative to find a nonlinear regularity between the test data and eliminate human factors to look forward to get a better prediction results.

\footnotetext{
Abbreviations

ANN: Artificial neural network; BP: Back propagation; EEMD: Ensemble empirical mode decomposition; ELM: Extreme learning machine; IEEMD: Improved ensemble empirical mode decomposition; IRR: Internet rate of return; NPV: Net present value; NPVR: Net present value rate; PC: Present cost; PT: Dynamic investment payback period; RBF: Radial basis function; ROI: Return on investment; WA: Wavelet analysis; WNN: Wavelet neural network
}

Funding

This work is supported by the National Science Foundation of China under Grant No. 61572012 and the Natural Science Fund of Hubei Province (No. 2017CFB598).

\begin{abstract}
Authors' contributions
$\mathrm{HL}$ proposed the framework of the General Data Evaluation System Based on Social Networks. Moreover, she also participated in the writing of this paper. XM carried out the economic evaluation of petroleum projects based on BP neural networks. CW carried out the simulation, and he wrote the initial version of this paper. FY contributed to optimizing the BP neural network. All authors read and approved the final manuscript.
\end{abstract}

\section{Competing interests}

The authors declare that they have no competing interests.

\section{Publisher's Note}

Springer Nature remains neutral with regard to jurisdictional claims in published maps and institutional affiliations.

\section{Author details}

${ }^{1}$ State Key Laboratory for Information Engineering in Surveying, Mapping and Remote Sensing, Wuhan University, Wuhan, China. ${ }^{2}$ School of Information Engineering, Hubei University of Economics, Wuhan, China. ${ }^{3}$ School of Optical-Electrical and Computer Engineering, University of Shanghai for Science and Technology, Shanghai, China. ${ }^{4}$ Zhongnan University of Economics and Law, Wuhan, China.

Received: 8 February 2018 Accepted: 11 April 2018

Published online: 08 May 2018

\section{References}

1. CM Bishop, Neural network and their applications. Revi. Scie. Instr 65, 18031832 (1994)

2. R Lippmann, An introduction to computing with neural nets. Acm Sigarch Comput. Arch. News 4(2), 4-22 (2003)

3. S Xie, Y Wang, Construction of Tree Network with Limited Delivery Latency in Homogeneous Wireless Sensor Networks[J]. Wireless Personal Communications 2014, 78(1):231-246

4. $\mathrm{M}$ Zhang, $\mathrm{H}$ Zhang, $\mathrm{Y}$ Huang, Sales forecasting based on ERP system through BP neural networks. Adv. Comput. Intell. 6382, 289-296 (2010)

5. D You, Technical economy and project economic evaluation (Tsinghua University Press, Beijing, 2009), pp. 62-90

6. Yunluo Yu, Wei Li, Deren Sheng, et al. A hybrid short-term load forecasting method based on improved ensemble empirical mode decomposition and back propagation neural network. J Zhejiang Universityence A, 2016, 17(2):101-114.

7. P Wang, G Zhao, X Yao, Applying back-propagation neural network to predict bus traffic. International Conference on Natural Computation, Fuzzy Systems and Knowledge Discovery. IEEE, 752-756 (2016)

8. Elons A S, Magdi D A, Elgendy M Y. A proposed model for predicting the drilling path based on hybrid Pso-Bp neural network. Sai Computing Conference. IEEE, 2016:148-155.

9. Al-Hnaity B, Abbod M. A novel hybrid ensemble model to predict FTSE100 index by combining neural network and EEMD. Control Conference. IEEE, 2015:3021-3028

10. Zhang D, Li H, Aziguli, et al. Predicting strip thickness of hot rolled sheet based on the combination of correlation analysis and extreme learning machine. Industrial Electronics and Applications. IEEE, 2015:477-482.

11. Purnawansyah, Haviluddin. Comparing performance of backpropagation and RBF neural network models for predicting daily network traffic. Makassar International Conference on Electrical Engineering and Informatics. IEEE, 2014, 1:166-169.

12. Zhang F, Dai H, Tang D, et al. Research on runoff predicting based on wavelet neural network conjunction model. International Conference on Information Science and Cloud Computing Companion. IEEE, 2014:841-845.

13. Khan S M U, Shaikh J M. Predicting students blood pressure by artificial neuron network: Facebook predict students blood pressure. Science and Information Conference. IEEE, 2014:430-437.

14. Mao M, Cao Y, Chang L. Improved fast short-term wind power prediction model based on superposition of predicted error. IEEE International Symposium on Power Electronics for Distributed Generation Systems. IEEE, 2014:1-6. 
15. Q Wang, Study on non-destructive detection method for fresh degree of eggs based on BP neural network. Trans. Chin. Soc. Agric. Machinery 37(1), 104-106 (2006)

16. Zhang J, Yang Y. BP neural network model based on the K-means clustering to predict the share price. Fifth International Joint Conference on Computational Sciences and Optimization. IEEE Comput Soc., 2012:181-184.

17. Zhang Z, Li B, Deng Z, et al. Research on movie box office forecasting based on Internet data. International Symposium on Computational Intelligence and Design. IEEE, 2016:83-86.

18. Gairaa K, Chellali F, Benkaciali S, et al. Daily global solar radiation forecasting over a desert area using NAR neural networks comparison with conventional methods. International Conference on Renewable Energy Research and Applications. IEEE, 2016:567-571.

19. Cao Y, Tian L, Zhao H. The application of BP neural net real-time data forecasting model used in home environment. IEEE International Conference on Cyber Technology in Automation, Control, and Intelligent Systems. IEEE, 2015:1486-1490.

20. HZ Chen, F Wang, CY Zheng, et al. Comprehensive Assessment of Bank Competitivity Based on BP Neural Network[J]. J. Shandong Inst. Min. Tech, 2003, 22(4):96-98.

21. Xue-Tong L, Wan-Li D. Oil price forecasting based on particle swarm neural network. Seventh International Conference on Measuring Technology and Mechatronics Automation. IEEE, 2015:712-715.

22. N Xiong, X Jia, LT Yang, et al., A distributed efficient flow control scheme for multirate multicast networks. IEEE Trans. Parallel Distributed Syst. 21(9), 1254-1266 (2010)

23. J Davis, An Introduction to Neural Networks[J]. J .Cogn. Neurosci. 1996, 8(4): 383-383.

24. Y Zhao, Research and Application on BP Neural Network Algorithm[C]// International Industrial Informatics and Computer Engineering Conference. 2015, 1:1444-1447.

25. N Xiong, AV Vasilakos, LT Yang, et al., Comparative analysis of quality of service and memory usage for adaptive failure detectors in healthcare systems. IEEE J. Selected Areas Commun. 27(4), 495-509 (2009)

26. LK Hansen, P Salamon, Neural network ensembles. IEEE Trans. Pattern Anal Mach Intell. 12(10), 993-1001 (2002)

27. Liu X. Analysis of the misunderstanding in investment project economic appraisal theory. International Conference on Business Management and Electronic Information. IEEE, 2011:196-198.

28. MJ Osborne, A resolution to the NPV-IRR debate? Q. Rev. Econ. Finance 50(2), 234-239 (2010)

29. Zhang H, Shi X. Economic evaluation of green buildings: A neural network approach. 2012.

30. Wang S.A research of the evaluation index of county economy index system based on the Zhongyuan economic zone. Management and Service Science (MASS), 2011 international conference on IEEE, 2011:1-4.

31. W Dong, Evaluation of undeveloped oil reserve and economic classification. Oil Gas Geol. 14(3), 251-257 (1993)

32. LI Min jie, Analysis of oil investment economic evaluation indicators overseas. J. Xian University Finance Econ, 2007, 20(1):76-79

33. H Wang, YX Wang, XS Zhang, The application of BP neural network in the risk analysis of project investment. Optimization Cap. Construction, 2007, 28(3):4-7.

34. Q Song, Q Jiang, Z Song, Economic evaluation of CO2-EOR industry chain in refineries. Acta Pet. Sin. 31(1), 119-125 (2015)

35. LA Shalabi, Z Shaaban, B Kasasbeh. Data mining: a preprocessing engine. J. Comput. Sci. 2(9), 735-739 (2006)

36. Shang G, Sun C. Application of BP neural network for predicting anode accuracy in ECM. International Symposium on Information Science and Engineering. IEEE, 2008:428-432.

37. Wang $P$, Zhao $T$, Fan $Y$, et al. Study of Chinese farmers income forecast model based on BP neural network. 2014.

38. YJ Ren, J Shen, J Wang, J Han, SY Lee, Mutual verifiable provable data auditing in public cloud storage. J. Internet Technol. 16(2), 317-323 (2015)

39. Shin-lke K. A two phase method for determining the number of neurons in the hidden layer of a 3-layer neural network. Sice Conference 2010, Proceedings of. IEEE, 2010:238-242.

40. B Gu, VS Sheng, Z Wang, et al., Incremental learning for v-support vector regression. Neural Netw. 67(C), 140-150 (2015)
41. EJ Teoh, KC Tan, C Xiang, Estimating the number of hidden neurons in a feedforward network using the singular value decomposition. IEEE Trans. Neural Netw. 17(6), 1623-1629 (2006)

42. Lee $\mathrm{T}$, Chung $\mathrm{F} \mathrm{L}$. Determining the number of hidden nodes by progressive training. Electronics Letters. 26(16), 1318-1320 (1990)

\section{Submit your manuscript to a SpringerOpen ${ }^{\circ}$ journal and benefit from:}

- Convenient online submission

- Rigorous peer review

- Open access: articles freely available online

- High visibility within the field

- Retaining the copyright to your article

Submit your next manuscript at $>$ springeropen.com 